\title{
Computer-Based Acoustic Detector for Determining the Type and Concentration of a Solution
}

\author{
Tariq M. Younes \\ Department Of Mechtronics Engineering \\ Faculty of Engineering Technology \\ Al Balq'a Applied University \\ 15008 Amman 11134 Jordan \\ tariqmog@bau.edu.jo
}

\begin{abstract}
The problem of determining the type and concentration of a solution is an important issue in food, medical and chemical industries. In this paper, a work effort has been made to explore the possibility of designing a computer-based acoustic detector to determine the type and concentration of a solution. Signal conditioning and processing was carried out by using labVIEW (G Language) VIs. Experimental results show that the produced acoustic signal frequency can be used as an informative parameter related with the type and concentration of a solution. Also, linear range of measurement is found of $10 \mathrm{~g} / 100 \mathrm{ml}$. Furthermore, acceptable results for common industrial applications are drawn with a maximum percentage error of $3 \%$ compared with the conventional approach. As a result, such detector can be integrated with control system in order to perform some control actions.
\end{abstract}

Keywords: standing wave, acoustic resonance, LabVIEW VI, type and concentration of solution.

\section{Introduction}

The problem of determining the type and concentration of a solution is still an important issue in solution mining and chemical processing [1]. Since undesirable containment of solute in water may cause noticeable change in environmental parameters which play a great role in human life and industry. For example, the solute content in liquefied food product is an important parameter in determining the value, stability, quality, and processibility of the product. The measurement and control of the amount of solute present is an important concern for food processors.

Another example is the utilization of ionic liquids which are finding widespread use as solvents in several applications, including biphasic catalysis, liquid-liquid extraction, and organometallic synthesis [2-4]. For those reasons, it is essential to have a fast and efficient method of determining the type of solute in water.

Several techniques and methods are in use, or have been investigated to determine the concentration of solution [5]. Electrical property of a solution is used to measure its concentration. An example is conductometry, which is based on measuring the presence of free ions (conductivity) of electrolyte solutions [6]. Infrared spectrophotometers have been utilized to measure moisture content, particularly in milk. The infrared spectrum of a sample is recorded by passing a beam of infrared through the sample [7]. Nuclear magnetic resonance is a technique, which is used to measure solids/liquids ratio and hence the solids content [8]. Other techniques such as chromatography, mass and photo spectrometers provide reliable measurement of concentration of a solution with high accuracy [9]. Nevertheless, these technologies are still fairly expensive. Selection of the right technique depends on the field of application where the measurement of concentration of solution is required. It depends also on the required accuracy, reliability of measurement, and the operating environment.

Ultrasound is also used for measuring the concentration of simple solutions. In this case, the speed of sound is used to characterize the structure of material. The behavior of traveled 
sound wave usually depends on the type of the medium i.e. it travels more rapidly through solids than liquids [10]. It was shown that the speed of sound in a fluid changes with concentration (solids content) of a given material, and ultrasound was utilized to measure the concentration of solution with good precision [11].

Acoustic Emission (AE) has been successfully applied in many fields of material sciences [12]. For example, $A E$ is a very attractive non intrusive technique for monitoring crystallization processes [13].

Acoustic methods based on creating an acoustic standing wave within a waveguide are also used to measure the concentration of substance in liquids and gases [14]. Computerized acoustic detectors $[14,15]$ were developed to measure concentration of gases and vapors. Such detectors were an integral part of gas chromatograph.

Acoustic field was also combined with thermo effect to build a simple analyzer [16] which was used to measure the concentration of water content in kerosene. The signal obtained by this analyzer was presented as a "chromatogram".

The literature review of existing measurement detectors or analyzers which are used to determine the type and concentration of a solution shows that this issue is still developing. This explains why research and development in this field is a viable continuous process. Determining the type and concentration and of a solution can be realized using different types of analyzers and detectors. The work described in this paper presents the possibility of using an acoustic measurement in order to determine the type and concentration of solution.

\section{Overview of the computer-based acoustic detector}

The block diagram of a computer-based acoustic detector (CBAD) is shown in Figure 1. This detector is used to determine the type of solution and its concentration.

It includes two heads of a stethoscope type $3 \mathrm{M}$ Littmann, acoustic receiver AR (microphone type HCM4530), acoustic transmitter AT ( speaker type TM-2A), air tubes, a chamber made of glass, sound card type Creative CT4810 and PC with LabVIEW software.

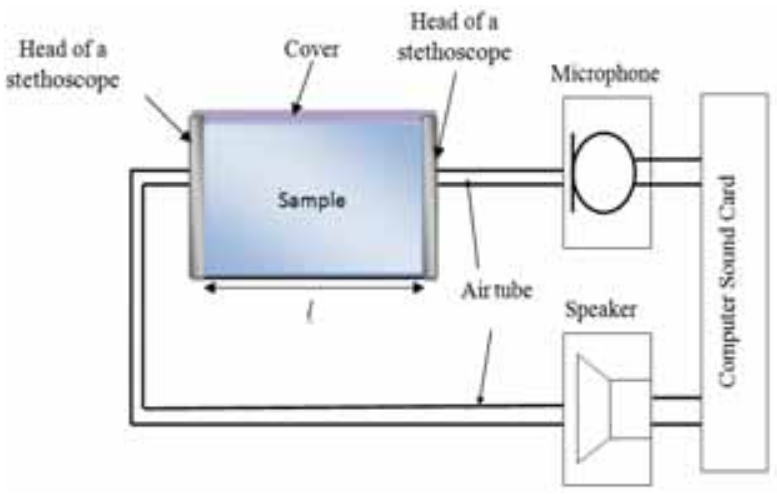

Figure 1. The block diagram of the computer-based acoustic detector.

The operating principle of the proposed detector is based on acoustic resonance when a standing wave is created in a chamber filled by a solution. The standing wave may be created using the principle of acoustic resonance, which is usually realized by using a speaker- microphone combination [17]. The sound card of PC is utilized in order to create an acoustic standing wave between the (AT) and (AR) which is transmitted through the sample under test. As a result, the speed of transmitted acoustic signal varies depending on the type of the medium filled in the chamber. This variation of speed is usually characterized by the frequency of created acoustic standing wave, when the distance between the two heads of stethoscope is constant.

\section{Mathematical model}

In section 2, we have mentioned that the speed of sound varies depending on the medium through which the sound wave passes. If the solution is an aqueous solution of $\mathrm{NaCl}$ or $\mathrm{KCl}$, the speed of sound is given by [18]

$$
C=\sqrt{\frac{E}{\rho}}
$$

where $C$ is the speed of sound, $E$ is the bulk modulus, and $\rho$ is the Density of media.

In $[14,19]$, it was shown that for aqueous solution, the speed of sound in a solution can be written as 


$$
C=\sqrt{\frac{\check{E}}{\check{\rho}}}
$$

where $\breve{\mathrm{E}}=\mathrm{E}_{\text {water }}+\mathrm{E}_{\text {solute }}$ and $\check{\rho}=\rho_{\text {water }}+\rho_{\text {solute }}$. The frequency of free resonance when a standing wave is generated within a chamber is given as

$$
\mathrm{f}=\frac{\mathrm{C}}{2 \mathrm{~L}}
$$

where $f$ is the frequency of generated standing wave in free resonance mode, $\mathrm{L}$ is the distance between two heads of stethoscope.

From (2) and (3), the frequency can be written as

$$
\begin{gathered}
f=\frac{\sqrt{\breve{E}}}{2 L}=\frac{\sqrt{\breve{E}}}{2 L} \cdot \sqrt{\frac{1}{\check{\rho}}} \\
\mathrm{f}=\frac{\sqrt{\breve{E}}}{2 \mathrm{~L}} \cdot \sqrt{\frac{1}{\check{\rho}}}=\mathrm{K} \sqrt{\frac{1}{\check{\rho}}}
\end{gathered}
$$

From (5), it is noted that the frequency is a function of density.

\section{Signal conditioning and processing}

Figure 2 shows the block diagram of LabVIEW program which is used to measure the frequency of the microphone input.

To measure the frequency, the signal from the microphone is fed to the MIC input of the sound card of PC. The sound card is configured to generate an acoustic standing wave in free resonance mode. In this case, the output of the $A R$ is fed to the sound card during which the signal is amplified and fed back to the AT. Then, this signal is measured using computer software. The signal acquisition and processing were realized using LabVIEW software [20-21]. In such experimental arrangement, one can measure, store, and log the data to the computer. Also, the use of measured variable for further display and control purposes is obtained. The detail description for each LabVIEW VIs used to build the block diagram is presented in appendix 1.

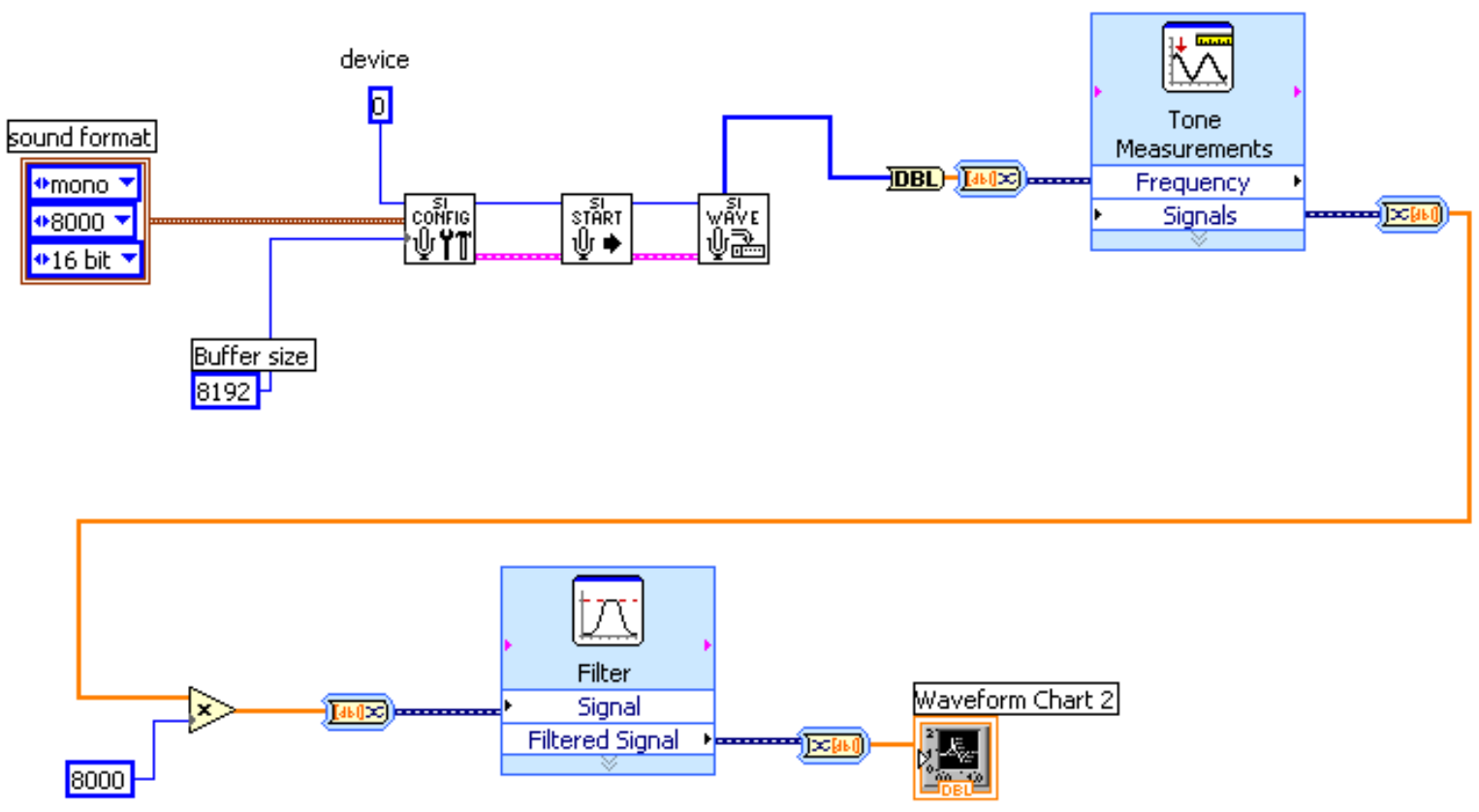

Figure 2. Block diagram of LabVIEW program. 


\section{Experimental results}

Aqueous solutions of sodium chloride and potassium chloride were used as samples under test. The resonance frequency of each medium as a function of concentration was determined and it was compared favorably with the conventional approach [22]. The frequency of sound was determined for aqueous solutions of sodium chloride and potassium chloride over applicable concentration range of $1-10 \mathrm{~g} / 100$ $\mathrm{ml}$ as shown in Table 1.

To represent experimental data, the method of data regression is used by utilizing Excel curve fitting. The linear regression model $y=a x+b$ is used to fit the regression model of the detector for both aqueous solutions of sodium chloride and potassium chloride as is shown in Figure 3.

From the experimental results and regression model, it is obvious that maximum relative error is equal to $3 \%$. Interestingly, it is worthy of noting that the environment influences as temperature; humidity and vibration have a minimum negligible effect on detector performance. That is due to that the chamber material is made of any isolating material with any wall thickness.

\begin{tabular}{|c|c|c|}
\hline \multirow{2}{*}{$\begin{array}{l}\text { Density of solution } \\
\qquad[\mathrm{g} / 100 \mathrm{ml}]\end{array}$} & \multicolumn{2}{|c|}{ Frequency, [Hz] } \\
\hline & $\mathrm{KCl}$ & $\mathrm{NaCl}$ \\
\hline 1 & 2300 & 2680 \\
\hline 2 & 2352 & 2700 \\
\hline 3 & 2380 & 2780 \\
\hline 4 & 2410 & 2830 \\
\hline 5 & 2430 & 2848 \\
\hline 6 & 2475 & 2890 \\
\hline 7 & 2510 & 2920 \\
\hline 8 & 2540 & 2958 \\
\hline 9 & 2590 & 3010 \\
\hline 10 & 2750 & 3045 \\
\hline
\end{tabular}

Table 1. Experimental results.

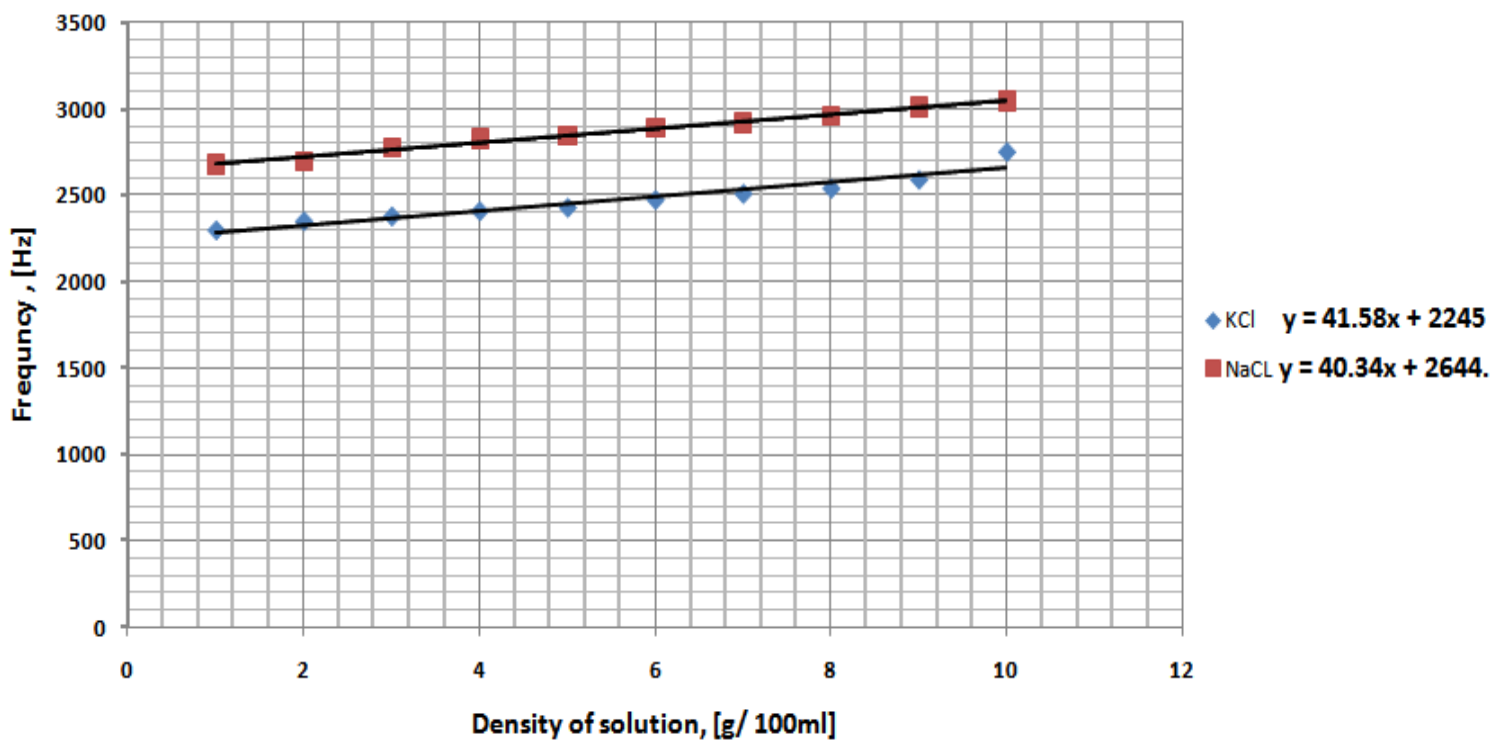

Figure 3. Measured frequency for aqueous solution of potassium chloride and sodium chloride. 


\section{Conclusions}

In this paper, a simple computerized measurement system was developed to determine the type and concentration of solution. Experimental results showed that it is possible to make use of the standing wave phenomenon for the design of acoustic detector which may be used to determine the concentration and the type of a solution. As a future work, it is possible to design an acoustic analyzer to determine the type and consternation of a solution using the proposed acoustic detector. Using such data acquisition system allows to log the measurement and to use it for further control purposes.

\section{Appendix 1}

\section{a) Sound Input CONFIGURE VI:}

The purpose of this $\mathrm{VI}$ is to configure a sound device for sound input operation as shown in Figure 4, the device is selected through the device number terminal, the selected number is 1 which is associated with the microphone in the LabVIEW, also the sound format terminal is fed by a constant controller.

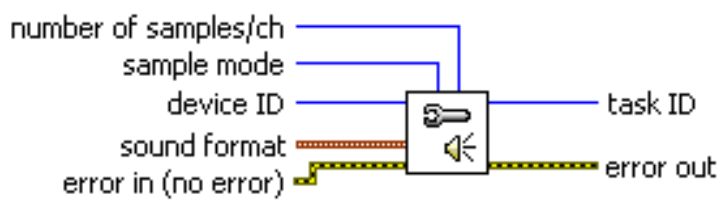

Figure 4. Sound input CONFIGURE VI.

b) Sound Input START VI:

This VI prompts the sound input device to begin accumulating incoming data as shown in Figure 5.

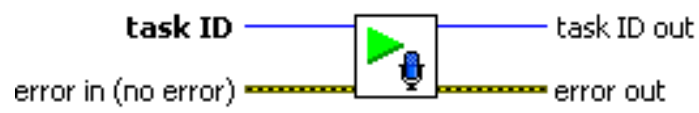

Figure 5. Sound input START VI c) Sound Input READ VI:

The SI READ VI is used to read data from the sound input device. If data has arrived in the device buffer; it returns the data after buffering. Otherwise, it waits until data arrives. The microphone signal wave graph is fed from the output of this VI. The symbol of the VI is shown in Figure 6.

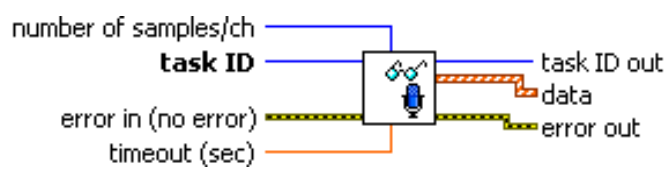

Figure 6. Sound input READ VI.

d) Tone Measurements VI:

The Tone Measurements VI shown in Figure 7 is used to measure the frequency of input signal and returns the value through its output terminal. The frequency numeric indicator in the front panel is fed from the output terminal of this VI.

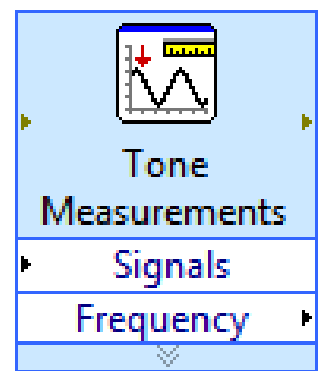

Figure 7. Tone measurements VI. 


\section{References}

[1] Sonic Method And Means For Determination Of Solution Concentrations, USA patent Number US3648513.

[2] J. Dupont R.F. de Souza, P.A.Z Suarez. Chem. Rev. 102 (2002) 3667

[3] P. J. Dyson, Appl. Organomet. Chem., 2002, 16, 495.

[4] Huddleston, J. G.; Willauer, H. W.; Swatloski, R. P.; Visser, A. E.; Rogers, R. D. "Room Temperature Ionic Liquids as Novel Media for 'Clean' Liquid-Liquid Extraction," Chem. Commun. 1998, 1765-1766.

[5] Bradley RL. 1994 Moisture and Total Solids Analysis. In: Nielsen SS, editor. Introduction to the Chemical Analysis of Foods. $1^{\text {st }}$ ed. Boston: Jones and Bartlett. P 93-111.

[6] Lyubov P Safonova and Arkadii M Kolker, Conductometry of electrolyte solutions, 1992 Russ. Chem. Rev. 61959

[7] Stuart, B. 2 Ed., Infrared Spectroscopy. ,KirkOthmer Encyclopedia of Chemical Technology, 2002.

[8] Basaran TK, Coupland JN, McClements DJ. 1999. Monitoring molecular diffusion of sucrose in xanthan solutions using velocity measurements. J Food Sci 64(1):125-128.

[9] Daniel C. Harris, Quantitative Chemical analysis, 8-th edition, 2010, Library of Congress Control Number: 2009943186.

[10] McClements DJ, Povey JW. 1987. Solid fat content determination using ultrasonic velocity measurements. Int J Food Sci Technol 22(6):491-499 .

[11] David P, Voge J. 1969. Propagation of waves. Oxford: Pergamon Press. 329 p. Del Grosso VA, Mader CW. 1972. Speed of sound in pure water. J Acoust Soc Am 52(5):1442-1446.A.

[12] Marec, J. Thomas, et R. El Guerjouma, "Damage characterization of polymerbased composite materials: Multivariable analysis and wavelet transform for clustering acoust. Emission data," Mech. Syst. \& Signal Proces., 22, 2008, 1441-64.
[13] Nesrine Gherras, Eric Serris And Gilles Févottea, Acoustic On-Line Monitoring Of Solution Crystallization Process In Pure And Impure Media, (International Symposium on Industrial Crystallization ISIC 18, Milano, Italy.

[14] Shebel A. Alsabbah \& Tariq Mughrabi. "Neural Network-Based Waveguide Acoustic Gas Detector". Proceeding of the 5th $t$ International Symposium on Mechatronics and its Applications (ISM08), IEEE Index. Amman, Jordan, May 27-29, 2008.

[15] Acoustic detector of gases and vapors, Tariq Younes, Ilyasov L. V. . Patent number 33822, 2003, Russian Federation.

[16] Tariq Mansour Al Mograbi, Thermoacoustic Analyzer for Water content Detection in Hydrocarbon Emulsion, Sensor Systems for Environmental Monitoring Conference held on the 14th October 2010 At The Royal Society of Chemistry Burlington House, London.

[17] Mohammad A.K. Alia and Tariq Al Mograbi, Investigation of An Acoustic Temperature Transducer and its Application for Heater Temperature Measurement, American Journal of Applied Sciences 4 (5): 294-299, 2007.

[18] Thomas D Rossing, Springer Handbook of Acoustics, 1-st Ed, 2007, XXIV, page 47.

[19] Green Book, 2nd ed., p. 42 PAC, 1996, 68, 957 (Glossary of terms in quantities and units in Clinical Chemistry (IUPAC-IFCC Recommendations 1996)) on page 979.

[20] Tariq Al Mograbi, Mohammad A. K. Alia, Mohammad Abuzalata, Design of an Acoustic Displacement Transducer, Sensors \& Transducers Journal, Vol. 112, Issue 1, January 2010, pp. 1-9.

[21] Johnson, G. W., LabVIEW Graphical Programming. Practical Applications in Instrumentation and Control.

[22] DelGRosso and E. J. Smura, . "Materials suitable for sound applicants: I. Ultrasonic velocities and impedance of selected liquids". MC Graw- Hill, Inc, USA, 1994.V. A Naval Research Laboratory, rep. 4191, 1953. 